\title{
A novel reflection on sleep disorders approach
}

Keywords: mood disorders, anxiety, depression, sleep disorders, psychological stress

\section{Opinion}

Mood disorders are likely to play a central role in the cognition of young adults, who do not have as many comorbidities or diagnoses of neurodegenerative diseases as the elderly population. The statistical population of recent published articles is overwhelmingly undergraduate and graduate students. Since the inclusion criteria are very broad, the sample under study is therefore very representative of the general population. In line with recent research, the results of Etindele Sosso confirmed the role of the environment in the occurrence of cognitive disorders; because they have identified during their investigations; a significant proportion of cognitive decline associated with the clinical history of the individual or their entourage. ${ }^{1-6}$ It is also proposed that reducing psychological stress would also reduce the onset or aggravation of cognitive impairment. According to their results, the continuous evaluation of brain performance while controlling the evolution of mood disorders (anxiety, depression and well-being) would contribute to a sensitive and accurate predictive model. ${ }^{7-11}$ Another conclusion would be that the living conditions marked by the working environment, the presence or not of a psychological or financial support; would facilitate the devastating effects of stress on cognition. ${ }^{12-18}$ In this context, sleep disorders would be indicators of cognitive failure well before the diagnosis of depression and anxiety. Cohort studies should be done to explore the effects on cognition of a stressful work environment such as customer service. The research of Etindele Sosso et al. ${ }^{8}$ confirmed the deleterious effect of permanent stress on the clinical course of sleep disorders and cognitive disorders. The current literature is not covering enough socio-demographic and environmental risks factors involved in sleep disorders. So, recent topics unrevealed by Etindele Sosso and his team are highly interesting, in the path they confirms the effect of psychological stress as a mediator of the effects of environmental stress on mental health.

\section{Conclusion}

Many investigations should be made to understand sleep disorders, and hopefully their mechanisms are linked to a plenty of symptoms and diseases emerging from lifestyles risk factors. Researches should be made in this way to support or rejects current findings on neurological deficits related to sleep.

\section{Funding details}

There is no implication of any funding organism in this research.

\section{Acknowledgements}

All authors mentioned above equally conceived, designed the study and wrote the final manuscript together.

\section{Conflict of interest}

The authors mentioned any conflict of interests for this study.
Volume I Issue 4 - 2017

\author{
Etindele Sosso Faustin Armel,' Nakamura \\ Okito, ${ }^{2}$ Bern Schmidt S, ${ }^{3}$ Mitsu Nakamura, ${ }^{4}$ \\ Muller G Hito ${ }^{5}$ \\ 'Department of Biological Sciences, University of Montreal, \\ Canada \\ ${ }^{2}$ Department Global Research, Ritsumeikan University, Japan \\ ${ }^{3}$ Department of Fundamental Neuroscience, University of \\ Lausanne, Switzerland \\ ${ }^{4}$ The Graduated University of Advanced Studies, Japan \\ ${ }^{5}$ Department of Psychology and Sports Science, Justus-Liebig \\ University, Germany
}

Correspondence: Etindele Sosso Faustin Armel, PhD, Department of Biological Sciences, University of Montreal, Canada, Email faustin.armel.etindele.sosso@umontreal.ca

Received: October 30, 2017 | Published: November 22, 2017

\section{References}

1. Etindele Sosso FA, Nakamura O, Nakamura M. Evaluation of combined effects of insomnia and stress on sleep quality and sleep duration. $J$ Neurol Neurosci. 2017;8(3):202.

2. Mitsu N, Nakamura O, Raouafi S, et al. Applications of the mental health profile of etindele questionnaire. SM J Sleep Disord. 2017;3(2):1012.

3. Etindele Sosso FA. Negative involvement of the working environment in the occurrence of cognitive disorders. Transl Biomed. 2017;8:2.

4. Raouafi S, Etindele Sosso FA. Cyberpsychology: video games as a perspective for cognitive training. Ment Health Addict Res. 2017;2(3):1-2.

5. Etindele Sosso FA. Sleep disorders and insomnia: effects on a young population. EC Psychology and Psychiatry. 2017;2(1):26-32.

6. Etindele Sosso FA. Neurocognitive game between risk factors, sleep and suicidal behaviour. Sleep Sci. 2017;10(1):41-46.

7. Etindele Sosso FA, Raouafi S. An overview of positive interaction between exercise and mental health. J Neurol Neurosci. 2017;8(4):215.

8. Etindele Sosso FA, Raouafi S. Brain disorders: correlation between cognitive impairment and complex combination. Ment Health Fam Med. 2016;12(2):215-222.

9. Etindele Sosso FA, Raouafi S. Appropriate sleep duration and physical activity modulate cognitive improvement. J Sleep Disor Treat Care. 2016;5:4.

10. Etindele Sosso FA, Hito MG, Bern SS. Basic activity of cortical neurons during somnolence induced by anesthesia. J Neurol Neurosci. 2017;8(4):203.

11. Etindele Sosso FA. Visual dot interaction with short-term memory. Neurodegener Dis Manag. 2017;7(3):183-190.

12. Etindele Sosso FA, Kaboré P. The African burden of mental health. J Ment Disord Treat. 2016;2:2. 
13. Pearson H. Genetics: what is a gene? Nature. 2006;441(7092):398-401.

14. Visscher PM, Hill WG, Wray NR. Heritability in the genomics eraconcepts and misconceptions. Nat Rev Genet. 2008;9(4):255-266.

15. Bijma P, Wade MJ. The joint effects of kin, multilevel selection and indirect genetic effects on response to genetic selection. J Evol Biol. 2008;21(5):1175-1188.

16. Milunsky. Genetic disorders and the fetus: diagnosis, prevention, and treatment. In: Aubrey editor. 5th ed. Baltimore, USA: Johns Hopkins University Press; 2004.
17. Wu R, Lin M. Functional mapping-how to map and study the genetic architecture of dynamic complex traits. Nat Rev Genet. 2006;7(3):229-237.

18. Ginn SL, Alexander IE, Edelstein ML, et al. Gene therapy clinical trials worldwide to 2012-an update. J Gene Med. 2013;15(2):65-77. 